\begin{tabular}{|c|l|}
\hline Title & Singularities of smooth mappings with patterns \\
\hline Author(s) & Saji, Kentaro; Takahashi, Masatomo \\
\hline Citation & Hokkaido University Preprint Series in Mathematics, 755, 1-19 \\
\hline Issue Date & 2005-11-16 \\
\hline DOI & 10.14943/83905 \\
\hline Doc URL & http://hdl.handle.net/2115/69563 \\
\hline Type & bulletin (article) \\
\hline File Information & pre755.pdf \\
\hline
\end{tabular}

Instructions for use 


\title{
Singularities of smooth mappings with patterns
}

\author{
Dedicated to Professor Takao Matumoto on his sixtieth birthday
}

\author{
Kentaro Saji and Masatomo Takahashi
}

November 16, 2005

\begin{abstract}
We study smooth mappings with patterns which given by certain divergence diagrams of smooth mappings. The divergent diagrams of smooth mappings can be regard as smooth mappings from manifolds with singular foliations. Our concerns are generic differential topology and generic smooth mappings with patterns. We give a generic semi-local classification of surfaces with singularities and patterns as an application of singularity theory.
\end{abstract}

\section{Introduction}

Divergent diagrams of map germs appear in several geometrical contexts, for example, vision theory, web geometry, differential equations, singularity theory (cf. [4]-[10, 13, 16, 17, 19, 20]) etc. In particular, Arnol'd [1] and Dufour [5, 6, 8] have investigated generic local classification of divergent diagrams. In order to study a smooth mapping with patterns, we consider a divergent diagram of smooth mappings. Let $M$ be a $m$-dimensional smooth compact manifold, $N$ and $P$ be $n$ and $p$-dimensional smooth manifolds respectively. Throughout this paper we shall assume that $p<m<n$. We consider the pair of smooth maps $(\mu, g): M \longrightarrow P \times N$, that is, a divergent diagram

$$
P \stackrel{\mu}{\longleftarrow} M \stackrel{g}{\longrightarrow} N
$$

Then we call the map $g$ and $\left.g\right|_{\mu^{-1}(c)}$ for each $c \in P$ a smooth map with patterns and the union of image of $g$ and of $\left.g\right|_{\mu^{-1}(c)}$ for each $c \in P$ a manifold with singularities and patterns.

2000 Mathematics Subject classification: $58 \mathrm{Kxx}, 53 \mathrm{~A} 05,53 \mathrm{~B} 25$

Key Words and Phrases. smooth mapping with patterns, manifold with singularities and patterns, divergent diagram, semi-local, singularity theory 
If $\mu$ is a submersion and $g$ is an embedding, then the image of $g$ and of $\left.g\right|_{\mu^{-1}(c)}$ for each $c \in P$ are smooth manifold and regular foliations. Also if $\mu$ is not a submersion, then the foliations is singular Haefliger foliations. For definition of singular Haefliger foliations, refer, for instance [15].

On the other hand, if $g$ is not an embedding, then there exists singularities, that is, selfintersections and singular values on image of $g$. In this paper, we consider that $\mu$ and $g$ might have singular points.

We give an equivalent relation among smooth maps with patterns or manifolds with singularities and patterns as a divergent diagram.

Definition 1.1. Let $(\mu, g): M \longrightarrow P \times N$ and $\left(\mu^{\prime}, g^{\prime}\right): M^{\prime} \longrightarrow P^{\prime} \times N^{\prime}$ be divergent diagrams. We say that $(\mu, g)$ and $\left(\mu^{\prime}, g^{\prime}\right)$ are equivalent as divergent diagrams if there exists diffeomorphisms $\kappa: P \longrightarrow P^{\prime}, \phi: M \longrightarrow M^{\prime}$ and $\Psi: N \longrightarrow N^{\prime}$ such that the diagram

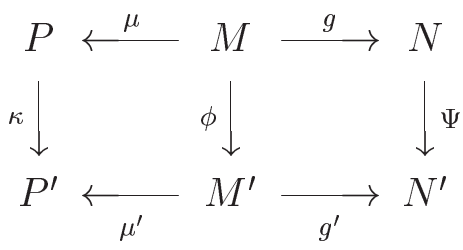

commutes.

If $(\mu, g)$ and $\left(\mu^{\prime}, g^{\prime}\right)$ are equivalent by above diffeomorphisms, then the diffeomorphism $\Psi$ takes the image of $g$ and of $\left.g\right|_{\mu^{-1}(c)}$ to image of $g^{\prime}$ and of $\left.g^{\prime}\right|_{\mu^{\prime-1}(\kappa(c))}$ for each $c \in P$ respectively.

We want to classificate manifolds with singularities and patterns via this equivalence relation. In Section 2, we shall prepare the notions and concepts of the basic singularity theory. In Section 3, we consider the surface cases and give a generic semi-local classification of surface with singularities and patterns (Theorems 3.1 and 3.2). In Section 4 and Section 5, we shall proof the Theorems 3.1 and 3.2 respectively.

All mappings, map germs and manifolds considered here are of class $C^{\infty}$.

The first-named author would like to express his sincere gratitude to Professor Takao Matumoto for his fruitful advice and constant encouragement. This work was partially supported by Research Fellowship of Japan Society for the Promotion of Science for Young Scientists.

\section{Basic notions and concepts}

In this section we review the basic notions and the concepts of viewpoint from the singularity theory for differential mappings (cf. [2, 11, 12, 18, 21, 22, 23]).

Let $M$ be a $m$-dimensional compact manifold and $N$ be a $n$-dimensional manifold. We denote that $C^{\infty}(M, N)$ as the set of maps from $M$ to $N$ and the topological set equipped with Whitney $C^{\infty}$-topology. Let $g: M \longrightarrow N$ be a map and $W$ be a submanifold of $N$. We say that 
$g$ is transverse to $W$ if for any $x \in M$, either

$$
(d g)_{x}\left(T_{x} M\right)+T_{g(x)} W=T_{g(x)} N
$$

or $g(x) \notin W$ holds. We present a multi-jet transversality theorem in multi-jet spaces which is useful for studying the generic property of smooth mappings. Define the map $g^{(r)}: M^{(r)} \longrightarrow N^{r}$ by the restriction of $g \times \cdots \times g(r$-times $): M^{r} \longrightarrow N^{r}$ to $M^{(r)}$, where

$$
M^{(r)}=\left\{\left(p_{1}, \ldots, p_{r}\right) \in M^{r} \mid p_{i} \neq p_{j} \text { for } 1 \leq i<j \leq r\right\} .
$$

Let $\pi_{M}: J^{k}(M, N) \longrightarrow M$ be the source map and $\pi_{M}^{r}: J^{k}(M, N)^{r} \longrightarrow M^{r}$ be the obvious projection. We call ${ }_{r} J^{k}(M, N)=\left(\pi_{M}^{r}\right)^{-1}\left(M^{(r)}\right)$ the $r$-fold $k$-jet space. The set $M^{(r)}$ is a manifold, since it is an open subset of $M^{r}$. Thus ${ }_{r} J^{k}(M, N)$ is an open subset of $J^{k}(M, N)^{r}$ and is also a manifold. We define multi $k$-jet extension of $g$ by ${ }_{r} j^{k} g: M^{(r)} \longrightarrow{ }_{r} J^{k}(M, N)$,

$$
{ }_{r} j^{k} g\left(x_{1}, \ldots, x_{r}\right)=\left(j^{k} g\left(x_{1}\right), \ldots, j^{k} g\left(x_{r}\right)\right) \text {. }
$$

Then by the multi-jet transversality theorem due to Mather [18], we have

Theorem 2.1. Let $M$ and $N$ be smooth manifolds with $W$ a submanifold of ${ }_{r} J^{k}(M, N)$. Let

$$
\mathcal{O}_{W}=\left\{g \in C^{\infty}(M, N) \mid{ }_{r} j^{k} g \text { is transverse to } W\right\} \text {. }
$$

Then $\mathcal{O}_{W}$ is a residual subset of $C^{\infty}(M, N)$. Moreover if $W$ is closed, then $\mathcal{O}_{W}$ is open.

Let $\Delta N^{r}=\left\{(y, \ldots, y) \in N^{r} \mid y \in N\right\}$ denotes the diagonal set. Then $g$ is a mapping with normal crossings if for every $r>1, g^{(r)}$ is transversal to $\Delta N^{r}$. As well-known result, we have the following proposition and lemma:

Proposition 2.2. (cf. [12, Proposition 3.2]) The set of mappings of $M$ into $N$ with normal crossings is a residual subset in $C^{\infty}(M, N)$.

We need to the process of the proof of the proposition.

Proof. Let $\beta^{r}:{ }_{r} J^{0}(M, N) \longrightarrow N^{r}$ be the multi-jet target mapping. Since $\beta^{r}$ is a submersion, $W^{r}=\left(\beta^{r}\right)^{-1}\left(\Delta N^{r}\right)$ is a submanifold of ${ }_{r} J^{0}(M, N)$. It is easy to check that $g$ is a mapping with normal crossings if and only if ${ }_{r} j^{0} g$ is transversal to $W^{r}$. Since codim $W^{r}=\operatorname{codim} \Delta N^{r}=$ $(r-1) \cdot \operatorname{dim} N$, for the number $r$ which satisfy the condition $r>n /(n-m),{ }_{r} j^{0} g$ is transversal to $W^{r}$ if and only if $g^{(r)}\left(M^{(r)}\right) \cap \Delta N^{r}=\emptyset$. Therefore for fixed a number $r_{0}$ which satisfy the above condition and $r_{0} j^{0} g$ is transversal to $W^{r_{0}}$, then for all $r>r_{0},{ }_{r} j^{0} g$ is transversal to $W^{r}$. We also consider the subset of $C^{\infty}(M, N)$,

$$
\mathcal{O}_{W^{r}}=\left\{g \in C^{\infty}(M, N) \mid{ }_{r} j^{0} g \text { is transverse to } W^{r}\right\}
$$

where $2 \leq r \leq r_{0}$. By the multi-jet transversal theorem, $\mathcal{O}_{W^{r}}$ is residual subset. Hence $\mathcal{O}=\cap_{r=2}^{r=r_{0}} \mathcal{O}_{W^{r}}$ is also residual subset. 
Let $V$ be a real vector space and let $H_{1}, \ldots, H_{r}$ be subspaces of $V$. Then $H_{1}, \ldots, H_{r}$ are said to be in general position if for every sequence of integers $i_{1}, \ldots, i_{s}$ with $1 \leq i_{1}<\cdots<i_{s} \leq r$,

$$
\operatorname{codim}\left(H_{i_{1}} \cap \cdots \cap H_{i_{s}}\right)=\operatorname{codim}\left(H_{i_{1}}\right)+\cdots+\operatorname{codim}\left(H_{i_{s}}\right) .
$$

Lemma 2.3. (cf. [12, Lemma 3.6]) Let $g: M \longrightarrow N$ be a mapping with normal crossings. For $q \in N$, let $\left\{p_{1}, \ldots, p_{r}\right\}$ be all distinct point of finite subset of $g^{-1}(q)$. Then the spaces $d g_{p_{1}}\left(T_{p_{1}} M\right), \ldots, d g_{p_{r}}\left(T_{p_{r}} M\right)$ are in general position as subspaces of $T_{q} N$.

If the spaces $d g_{p_{1}}\left(T_{p_{1}} M\right), \ldots, d g_{p_{r}}\left(T_{p_{r}} M\right)$ are in general position as subspaces of $T_{q} N$, then we say that image of $g_{1}, \ldots, g_{r}$ are general position in $N$, for short. Here we denote $g_{i}$ by the map germ of $g$ at $p_{i}(i=1, \ldots, r)$.

Now we consider the divergent diagram $(\mu, g): M \longrightarrow P \times N$, where $P$ is a $p$-dimensional manifold. In order to describe the genericity of our situation, we shall also prepare a multi-jet transversality theorem for divergent diagrams. Let ${ }_{r} J^{\ell}(M, P \times N)$ denote the multi-jet space from $M$ to $P \times N$ of multiplicity $r$ and order $\ell$. For a map $(\mu, g): M \longrightarrow P \times N$, we denote by ${ }_{r} j^{\ell}(\mu, g): M^{(r)} \longrightarrow{ }_{r} J^{\ell}(M, P \times N)$ the multi $\ell$-jet extension of $(\mu, g)$.

Then by the multi-jet transversality theorem, we also have

Theorem 2.4. Let $M, N$ and $P$ be smooth manifolds. For any submanifold $Q \subset_{r} J^{\ell}(M, P \times N)$, the set

$$
T_{Q}=\left\{(\mu, g) \in C^{\infty}(M, P \times N) \mid{ }_{r} j^{\ell}(\mu, g) \text { is transverse to } Q\right\}
$$

is a residual subset of $C^{\infty}(M, P \times N)$. If $Q$ is closed, then $T_{Q}$ is an open set.

By definition of transversality, we have the following corollary:

Corollary 2.5. Let $Q$ be a submanifold of $r J^{\ell}(M, P \times N)$ and $\operatorname{codim} Q>r \cdot \operatorname{dim} M$. Then the set

$$
T_{Q}=\left\{(\mu, g) \in C^{\infty}(M, P \times N) \mid{ }_{r} j^{\ell}(\mu, g)\left(M^{(r)}\right) \cap Q=\emptyset\right\}
$$

is residual subset of $C^{\infty}(M, P \times N)$.

We now consider the relationship between $T_{Q}$ and $\mathcal{O}_{W}$. Let $\pi:_{r} J^{k}(M, P \times N) \longrightarrow_{r} J^{k}(M, N)$ be a canonical projection and $W$ be a submanifold of ${ }_{r} J^{k}(M, N)$. Then $\pi^{-1}(W)$ is also a submanifold of ${ }_{r} J^{k}(M, P \times N)$ and it is easy to check that ${ }_{r} j^{k} g$ is transversal to $W$ if and only if ${ }_{r} j^{k}(\mu, g)$ is transversal to $\pi^{-1}(W)$. Hence $g \in \mathcal{O}_{W}$ if and only if $(\mu, g) \in T_{\pi^{-1}(W)}$. As a result of multi-jet transversality theorem, we have the following lemma:

Lemma 2.6. Under the same notation of previous paragraph, we have $\mathcal{O}_{W}$ is a residual subset of $C^{\infty}(M, N)$ if and only if $T_{\pi^{-1}(W)}$ is a residual subset of $C^{\infty}(M, P \times N)$.

If we assume that $g$ is a mapping with normal crossings, then $g^{(r)}\left(M^{(r)}\right) \cap \Delta N^{r}=\emptyset$ for any number $r$ such that $r>n /(n-m)$ by Proposition 2.2. Therefore for any $p \in M$, the number of inverse image $\sharp g^{-1}(g(p))$ is less than or equal to $n /(n-m)$. 
Let $(\mu, g): M \longrightarrow P \times N$ be a divergent diagram. In this paper, we consider the generic mappings with patterns. Hence we may assume that $g$ is a mapping with normal crossings. By Lemma 2.6, for any point $p \in M$, we denote all distinct point of the inverse image of $p$ by $\left\{p_{1}, \ldots, p_{r}\right\}$ where $r \leq n /(n-m)$ is some natural number. Furthermore, by Lemma 2.3, image of $g_{1}, \ldots, g_{r}$ are general position in $(N, g(p))$ where map germ $g_{i}$ is the map germ $g$ at $p_{i}$ for $i=1, \ldots, r$. We also denote map germ $\mu_{i}$ as the map germ $\mu$ at $p_{i}$ for $i=1, \ldots, r$ (see, Figure 1 ). If $(\mu, g)$ and $\left(\mu^{\prime}, g^{\prime}\right)$ are equivalent, then $\sharp g^{-1}(g(p))$ and $\sharp g^{\prime-1}\left(g^{\prime}(\phi(p))\right)$ are same for any $p \in M$ and there exists diffeomorphism germs $\kappa_{i}:\left(P, \mu_{i}\left(p_{i}\right)\right) \longrightarrow\left(P^{\prime}, \mu_{\sigma(i)}^{\prime}\left(p_{\sigma(i)}^{\prime}\right)\right), \phi_{i}:\left(M, p_{i}\right) \longrightarrow$ $\left(M^{\prime}, p_{\sigma(i)}^{\prime}\right)$ and $\Psi:\left(N, g_{i}\left(p_{i}\right)\right) \longrightarrow\left(N^{\prime}, g_{\sigma(i)}^{\prime}\left(p_{\sigma(i)}^{\prime}\right)\right)$ such that $\kappa_{i} \circ \mu_{i}=\mu_{\sigma(i)}^{\prime} \circ \phi_{i}, \Psi \circ g_{i}=g_{\sigma(i)}^{\prime} \circ \phi_{i}$ for $i=1, \ldots, r$ where $\sigma$ is a permutation (i.e., $\sigma$ belong to the symmetry group $S_{r}$ ). In this case, we say that $\left(\mu_{i}, g_{i}\right)$ and $\left(\mu_{i}^{\prime}, g_{i}^{\prime}\right)$ are equivalent (see, Figure 2).

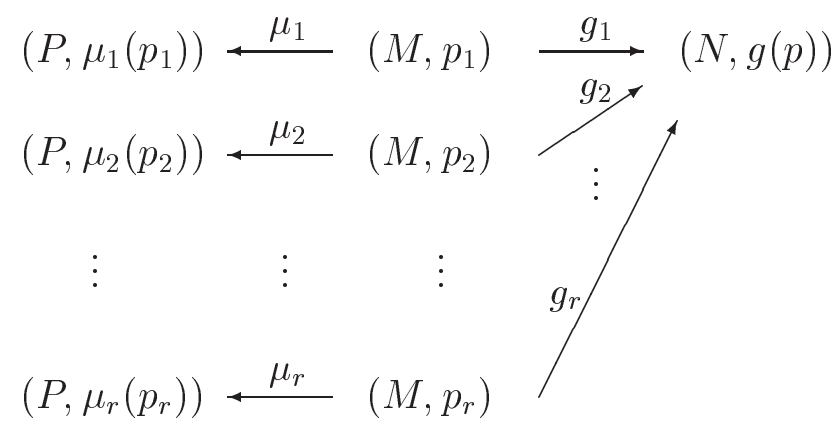

Figure 1: Multi-germs of divergent diagram

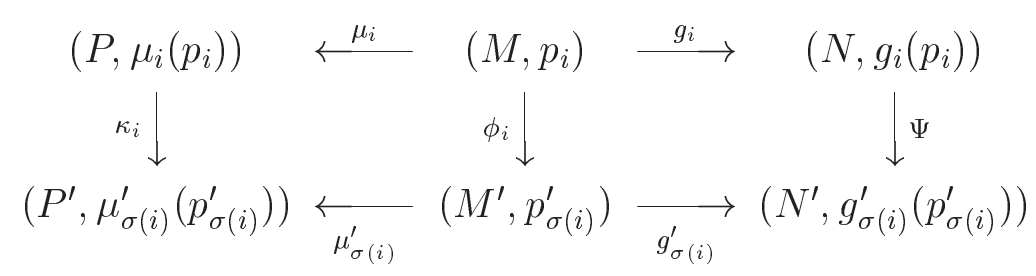

Figure 2: Equivalence of multi-germs of divergent diagrams

\section{Surfaces with patterns}

In this section we strict to surfaces cases. Let $M$ be a 2-dimensional compact manifold, $N$ be a 3-dimensional manifold and $P$ be a 1-dimensional manifold. Denote by $\mathcal{E}_{\left(x_{1}, \ldots, x_{n}\right)}$ the ring of all function germs on $\mathbb{R}^{n}$ at 0 with coordinates $\left(x_{1}, \ldots, x_{n}\right)$ and denote by $\mathcal{M}_{\left(x_{1}, \ldots, x_{n}\right)}$ the unique maximal ideal of $\mathcal{E}_{\left(x_{1}, \ldots, x_{n}\right)}$.

We shall state our theorems of a genericity, characterizations and their normal forms for surfaces with singularities and patterns. 
Theorem 3.1. There exists a residual subset $\mathcal{O}$ in $C^{\infty}(M, P \times N)$ such that for any $(\mu, g) \in \mathcal{O}$ and for any $p \in M$, the number of inverse image $r=\sharp g^{-1}(g(p)) \leq 3$ and $(\mu, g)$ is one of germ of the following type:

In the case of $r=1$,

(I) $\mu_{1}$ is a submersion germ and $g_{1}$ is an immersion germ.

(II) $\mu_{1}$ is a Morse type and $g_{1}$ is an immersion germ.

(III) $\mu_{1}$ is a submersion germ, $g_{1}$ is right-left equivalent to the Whitney umbrella, $\mu \circ c$ is a submersion germ and the pair $\left(\mu_{1}, g_{1}\right):\left(\mathbb{R}^{2}, 0\right) \longrightarrow\left(\mathbb{R} \times \mathbb{R}^{3}, 0\right)$ is an immersion germ. Here $c$ is the double point curve of $g_{1}$.

In the case of $r=2$,

$(\mathrm{I}, \mathrm{I})_{1} \quad\left(\mu_{1}, g_{1}\right)$ and $\left(\mu_{2}, g_{2}\right)$ are both of type $(\mathrm{I}), g_{1}$ and $g_{2}$ are mappings with normal crossings and $\mu_{1} \circ c_{12}$ and $\mu_{2} \circ c_{21}$ are submersion germs. Here $c_{i j}$ is the double point curve of $g_{i}$ and $g_{j}$ through $p_{i}$.

$(\mathrm{I}, \mathrm{I})_{2} \quad\left(\mu_{1}, g_{1}\right)$ and $\left(\mu_{2}, g_{2}\right)$ are both of type $(\mathrm{I}), g_{1}$ and $g_{2}$ are mappings with normal crossings, $\mu_{1} \circ c_{12}$ is a Morse function germ and $\mu_{2} \circ c_{21}$ is a submersion germ. Here $c_{i j}$ is the double point curve of $g_{i}$ and $g_{j}$ through $p_{i}$.

In the case of $r=3$,

(I,I,I) $\left(\mu_{1}, g_{1}\right),\left(\mu_{2}, g_{2}\right)$ and $\left(\mu_{3}, g_{3}\right)$ are all of type (I), $g_{1}, g_{2}$ and $g_{3}$ are mappings with normal crossings and $\mu_{i} \circ c_{i j}(i j=12,21,13,31,23$ and 32$)$ are all submersion germs. Here $c_{i j}$ is the double point curve of $g_{i}$ and $g_{j}$ through $p_{i}$.

Theorem 3.2. There exists a residual subset $\mathcal{O}$ in $C^{\infty}(M, P \times N)$ such that for any $(\mu, g) \in \mathcal{O}$ and for any $p \in M$, the number of inverse image $r=\sharp g^{-1}(g(p)) \leq 3$ and multi-germs of the each type are equivalent to one of the following type:

In the case of $r=1$,

(I) $\mu_{1}=y_{1}, g_{1}=\left(x_{1}, y_{1}, 0\right)$.

$(\mathrm{II})_{ \pm} \quad \mu_{1}=x_{1}^{2} \pm y_{1}^{2}, g_{1}=\left(x_{1}, y_{1}, 0\right)$

(III) $\mu_{1}=x_{1}+\alpha\left(x_{1}^{2}\right)+y_{1}, g_{1}=\left(x_{1}^{2}, y_{1}, x_{1} y_{1}\right)$, where $\alpha \in \mathcal{M}_{x_{1}}$.

In the case of $r=2$,

$$
\begin{aligned}
(\mathrm{I}, \mathrm{I})_{1} \quad \mu_{1} & =y_{1}, g_{1}=\left(x_{1}, y_{1}, 0\right) \\
& \mu_{2}=x_{2}, g_{2}=\left(0, x_{2}, y_{2}\right) . \\
(\mathrm{I}, \mathrm{I})_{2} \quad \mu_{1} & =x_{1}+y_{1}^{2}, g_{1}=\left(x_{1}, y_{1}, 0\right) \\
& \mu_{2}=x_{2}, g_{2}=\left(0, x_{2}, y_{2}\right) .
\end{aligned}
$$

In the case of $r=3$, 


$$
\begin{aligned}
& \mu_{1}=x_{1}+y_{1}, g_{1}=\left(x_{1}, y_{1}, 0\right) \\
& \mu_{2}=x_{2}+y_{2}, g_{2}=\left(0, x_{2}, y_{2}\right) \\
& \mu_{3}=x_{3}+y_{3}+\beta\left(x_{3}, y_{3}\right), g_{3}=\left(x_{3}, 0, y_{3}\right), \text { where } \beta \in \mathcal{M}_{\left(x_{3}, y_{3}\right)} .
\end{aligned}
$$

Here we denote by $\left(x_{i}, y_{i}\right)$ the local coordinate in the source space of $\mu_{i}$ and $g_{i}$ for $i=1,2,3$. We call $\alpha$ in (III) and $\beta$ in (I,I,I) functional moduli.

In order to understand our classification of surfaces with singularities and patterns, let us describe the image of $g$ and of $\left\{\left.g\right|_{\mu^{-1}(c)}\right\}_{c \in(\mathbb{R}, 0)}$ for each type (see, Figure 3).

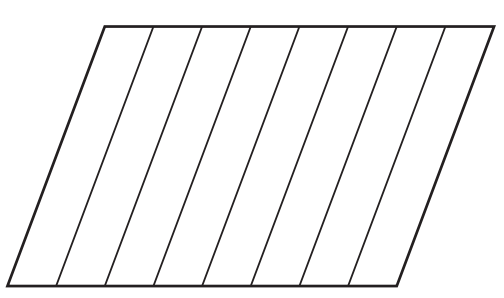

(I)

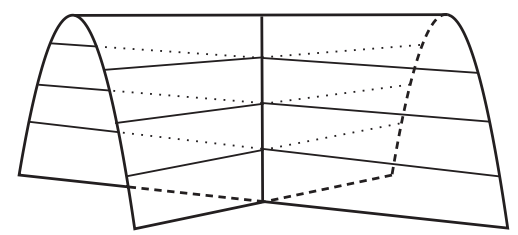

(III) where $\alpha=0$

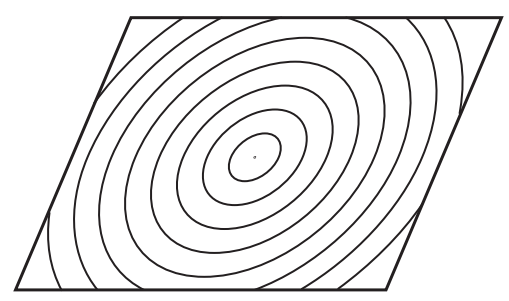

(II) +

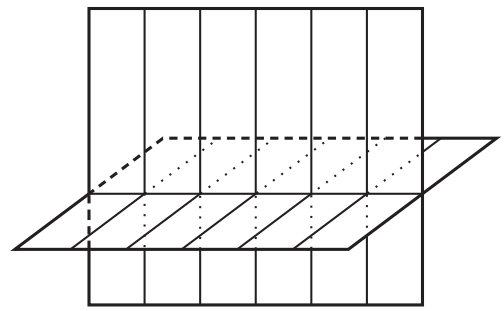

$(\mathrm{I}, \mathrm{I})_{1}$

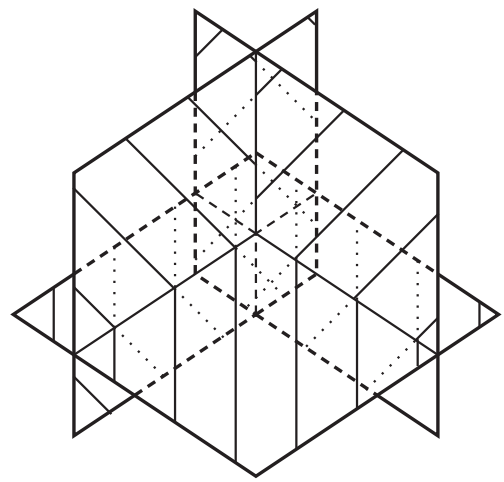

(I,I,I) where $\beta=0$

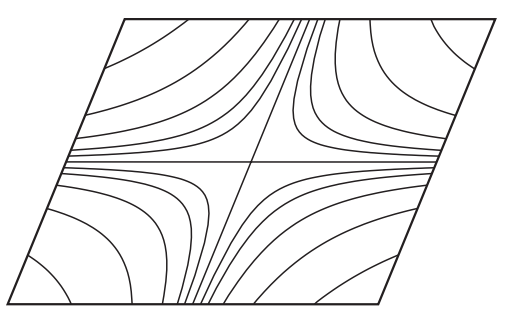

(II)

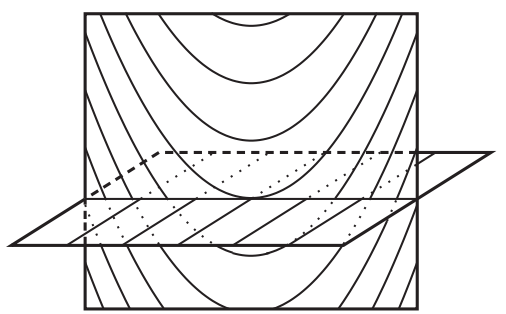

$(\mathrm{I}, \mathrm{I})_{2}$

Figure 3: Generic local examples of surfaces with singularities and patterns

We remark that in $[1,5]$ it have been considered the generic classification of the divergent diagram of type

$$
(\mathbb{R}, 0) \stackrel{\mu}{\longleftarrow}\left(\mathbb{R}^{2}, 0\right) \stackrel{g}{\longrightarrow}\left(\mathbb{R}^{2}, 0\right) .
$$

It is corresponding to the family of plane curves when $\mu$ is a submersion germ. Also in [16] Kurokawa has studied the generic semi-local classification of such divergent diagrams. In this 
sense, Theorem 3.2 give a generic semi-local classification of family of space curves when $\mu$ is a submersion.

On the other hand, in $[6,7,9,13]$ they have considered the topological rigidity and characterization of functional modulies. We are interested in a characterization and a geometric meaning of the functional modulies. Furthermore, we would like to consider topological properties of manifolds with singularities and patterns like as Izumiya-Marar [14]. These are future problems for us.

\section{Proof of Theorem 3.1}

First we consider the case of $r=1$. If $p \in M$ be a point that $g$ at $p$ is a Whitney umbrella germ, then we denote by $c(t)=\left(c_{1}(t), c_{2}(t)\right)$ a double point curve on $M$ such that $c(0)=p$.

We now consider the following subsets of $J^{2}(2,4)_{(p)}$ :

$$
\begin{aligned}
& \Sigma_{1}=\left\{j^{2}(\mu, g)(p) \mid \mu \text { is singularity and not Morse type at } p\right\}, \\
& \Sigma_{2}^{1}=\left\{j^{2}(\mu, g)(p) \mid \operatorname{corank}(d g(p))=1\right\}, \Sigma_{2}^{2}=\left\{j^{2}(\mu, g)(p) \mid \operatorname{corank}(d g(p))=2\right\}, \\
& \Sigma_{3}^{1}=\left\{j^{2}(\mu, g)(p) \mid \operatorname{corank} d(\mu, g)(p)=1\right\}, \Sigma_{3}^{2}=\left\{j^{2}(\mu, g)(p) \mid \operatorname{corank} d(\mu, g)(p)=2\right\} \text { and } \\
& \Sigma_{4}=\left\{j^{2}(\mu, g)(p) \mid g \text { at } p \text { is Whitney umbrella and }\left.(d / d t)(\mu \circ c)\right|_{t=0}=0\right\} .
\end{aligned}
$$

Then $\Sigma_{1}$ is a closed submanifold with codimension $3, \Sigma_{2}^{1}$ is a closed submanifold with codimension $2, \Sigma_{2}^{2}$ is a closed submanifold with codimension $6, \Sigma_{3}^{1}$ is a closed submanifold with codimension 3 and $\Sigma_{3}^{2}$ is a closed submanifold with codimension 8 (cf. [3]). We now show that $\Sigma_{4}$ is closed submanifold with codimension 3 . Let $(x, y)$ be a local coordinate of a neighborhood $U$ at $p$. Since

$$
\frac{d \mu \circ c}{d t}(0)=\frac{\partial \mu}{\partial x}(p) \cdot \frac{\partial c_{1}}{\partial t}(0)+\frac{\partial \mu}{\partial y}(p) \cdot \frac{\partial c_{2}}{\partial t}(0)
$$

and always $c$ is an immersion, $\Sigma_{4}$ is a submanifold with codimension 3 . We put $\widetilde{\Sigma_{4}}=\mathbb{R}^{2} \times \mathbb{R} \times$ $\mathbb{R}^{3} \times \Sigma_{4}$. Since $\widetilde{\Sigma_{4}}$ is an invariant set with respect to equivalence of divergent diagrams, we can extend to submanifold $\overline{\Sigma_{4}}$ with codimension 3 in the jet space $J^{2}(M, P \times N)$. We also extend to the submanifolds $\overline{\Sigma_{1}}, \overline{\Sigma_{2}^{1}}, \overline{\Sigma_{2}^{2}}, \overline{\Sigma_{1}^{3}}$ and $\overline{\Sigma_{2}^{3}}$ in the jet space $J^{2}(M, P \times N)$.

From the multi-jet transversality theorem,

$$
\mathcal{O}_{1}=\left\{(\mu, g) \in C^{\infty}(M, N \times P) \mid j^{2}(\mu, g) \text { is transverse to } \overline{\Sigma_{1}}, \overline{\Sigma_{2}^{1}}, \overline{\Sigma_{2}^{2}}, \overline{\Sigma_{1}^{3}}, \overline{\Sigma_{2}^{3}} \text { and } \overline{\Sigma_{4}}\right\}
$$

is residual subset of $C^{\infty}(M, N \times P)$.

By the characterization of the Whitney umbrella, it holds that $j^{2}(\mu, g)$ is transverse to $\overline{\Sigma_{2}^{1}} \cup \overline{\Sigma_{2}^{2}}$ implies $g$ at $p$ is right-left equivalent ( $\mathcal{A}$-equivalent) to the Whitney umbrella, namely, there exists diffeomorphism germs $h$ at $p$ and $k$ at $g(p)$ such that $k \circ g \circ h^{-1}(x, y)=\left(x^{2}, y, x y\right)$. 
Therefore, for any $p \in M$, if $\sharp g^{-1}(g(p))=1$, then $(\mu, g) \in \mathcal{O}_{1}$ satisfies the following conditions:

(1) $\mu$ at $p$ is a submersion germ or a Morse type.

(2) $g$ at $p$ is an immersion germ or a Whitney umbrella.

(3) $(\mu, g)$ at $p$ is an immersion germ.

(4) If $g$ at $p$ is the Whitney umbrella, $\mu$ is an immersion on the double point curve of $g$. Hence we have Theorem 3.1 when the case of $r=1$.

In the case of $r=2$. We put $g^{-1}(g(p))=\left\{p_{1}, p_{2}\right\}$. Under this notation, we have the following lemma:

Lemma 4.1. There exists a residual subset $\mathcal{O}_{2} \subset C^{\infty}(M, P \times N)$ such that for any $(\mu, g) \in \mathcal{O}_{2}$ and $p \in M$, if $\sharp g^{-1}(g(p))=2$, then map germs $(\mu, g)$ at $p_{1}$ and $p_{2}$ satisfy the conditions $(\mathrm{I}, \mathrm{I})_{1}$ or $(\mathrm{I}, \mathrm{I})_{2}$ of Theorem 3.1 .

We prove the Lemma by the following way: First, we assume that the two sheets near $g(p)$ intersect transversally. Then we have the regular double curves $c_{12}$ and $c_{21}$ on the source manifolds. It is sufficient that we prove the sets consist of $(\mu, g)$ such that satisfies the assumption, moreover $\mu_{1} \circ c_{12}$ and $\mu_{2} \circ c_{21}$ are both of immersion germs or one of them is an immersion and the another has Morse type critical point is a residual set. To prove this, we calculate first and second derivatives of $c_{12}$ and $c_{21}$ using the implicit function theorem. Curves $c_{12}$ and $c_{21}$ are characterized by the equation $g_{1}\left(x_{1}, y_{1}\right)-g_{2}\left(x_{2}, y_{2}\right)=0$ on the coordinates $\left(x_{1}, y_{1}\right)$ near $p_{1}$ and coordinates $\left(x_{2}, y_{2}\right)$ near $p_{2}$. The implicit function theorem works and one can get the derivatives of $c_{12}$ and $c_{21}$. Finally, we use the standard method of singularity theory, prove the set is residual.

Proof of Lemma 4.1. We take open neighborhoods $U_{1}$ at $p_{1}$ and $U_{2}$ at $p_{2} V$ at $f\left(p_{1}\right)=f\left(p_{2}\right)$, local coordinates $\left(x_{1}, y_{1}\right)$ on $U_{1}$ and $\left(x_{2}, y_{2}\right)$ on $U_{2}$. Denote $g_{1}=\left(g_{11}, g_{12}, g_{13}\right)$ (respectively, $\left.g_{2}=\left(g_{21}, g_{22}, g_{23}\right)\right)$ by map germs $g$ at $p_{1}$ (respectively, at $p_{2}$ ) with respect to the coordinates. First, we put

$$
S_{1}=\left\{{ }_{2} j^{2}(\mu, g)(p) \in{ }_{2} J^{2}(2,4)_{(p)} \mid d g_{1}\left(p_{1}\right)=0\right\}
$$

and

$$
S_{2}=\left\{{ }_{2} j^{2}(\mu, g)(p) \in{ }_{2} J^{2}(2,4)(p) \mid d g_{2}\left(p_{2}\right)=0\right\} .
$$

Then $S_{1}$ and $S_{2}$ are closed algebraic subset with codimension 2. Note that $X={ }_{2} J^{2}(2,4)_{(p)} \backslash$ $S_{1} \cup S_{2}$ is an open submanifold of ${ }_{2} J^{2}(2,4)_{(p)}$. Second, we put

$$
\begin{aligned}
& S_{21}=\left\{{ }_{2} j^{2}(\mu, g)(p) \in X \mid \Delta_{12}^{1}\left(p_{1}, p_{2}\right)=0\right\}, S_{22}=\left\{{ }_{2} j^{2}(\mu, g)(p) \in X \mid \Delta_{12}^{2}\left(p_{1}, p_{2}\right)=0\right\}, \\
& S_{23}=\left\{{ }_{2} j^{2}(\mu, g)(p) \in X \mid \Delta_{21}^{1}\left(p_{1}, p_{2}\right)=0\right\}, S_{24}=\left\{{ }_{2} j^{2}(\mu, g)(p) \in X \mid \Delta_{21}^{2}\left(p_{1}, p_{2}\right)=0\right\},
\end{aligned}
$$


where

$$
\begin{aligned}
& \Delta_{i j}^{k}\left(x_{1}, y_{1} ; x_{2}, y_{2}\right)=(-1)^{k} \operatorname{det}\left(\operatorname{sign}(j-i) \cdot \frac{\partial g_{i}}{\partial x_{i}}\left(x_{i}, y_{i}\right) \quad \frac{\partial g_{i}}{\partial y_{i}}\left(x_{i}, y_{i}\right) \quad \frac{\partial g_{j}}{\partial x_{j}}\left(x_{j}, y_{j}\right) \quad \frac{\partial g_{j}}{\partial y_{j}}\left(x_{j}, y_{j}\right)\right)^{\breve{k}} \\
& =(-1)^{k} \operatorname{det}\left(\begin{array}{cccc}
\operatorname{sign}(j-i) \cdot \frac{\partial g_{i 1}}{\partial x_{i}} & \frac{\partial g_{i 1}}{\partial y_{i}} & \frac{\partial g_{j 1}}{\partial x_{j}} & \frac{\partial g_{j 1}}{\partial y_{j}} \\
\operatorname{sign}(j-i) \cdot \frac{\partial g_{i 2}}{\partial x_{i}} & \frac{\partial g_{i 2}}{\partial y_{i}} & \frac{\partial g_{j 2}}{\partial x_{j}} & \frac{\partial g_{j 2}}{\partial y_{j}} \\
\operatorname{sign}(j-i) \cdot \frac{\partial g_{i 3}}{\partial x_{i}} & \frac{\partial g_{i 3}}{\partial y_{i}} & \frac{\partial g_{j 3}}{\partial x_{j}} & \frac{\partial g_{j 3}}{\partial y_{j}}
\end{array}\right)^{\breve{k}}
\end{aligned}
$$

Here, $A^{\breve{k}}$ means a matrix which removed the $k$-th column from the matrix $A$ and $\operatorname{sign}(\tau)$ takes 1 (respectively -1) when $\tau$ is positive (respectively negative). Then $S_{21} \cap S_{22}$ and $S_{23} \cap S_{24}$ are submanifolds with codimension 2 . In fact, we define maps $\zeta_{i_{1}, \ldots, i_{6}}: X \longrightarrow \mathbb{R}^{2}\left(i_{1}, \ldots, i_{6}=1,2\right)$ by

$$
\zeta_{i_{1}, \ldots, i_{6}}\left({ }_{2} j^{2}(\mu, g)\left(x_{1}, y_{1} ; x_{2}, y_{2}\right)\right)=\left(\Delta_{i_{1} i_{2}}^{i_{3}}\left(x_{1}, y_{1} ; x_{2}, y_{2}\right), \Delta_{i_{4} i_{5}}^{i_{6}}\left(x_{1}, y_{1} ; x_{2}, y_{2}\right)\right) \text {. }
$$

Then $S_{21} \cap S_{22}=\left(\zeta_{121122}\right)^{-1}(0,0)$ holds and we calculate the derivatives of $\zeta_{121122}$ with respect to $\partial g_{11} / \partial y_{1}, \partial g_{12} / \partial y_{1}, \partial g_{13} / \partial y_{1}, \partial g_{11} / \partial x_{1}, \partial g_{12} / \partial x_{1}$ and $\partial g_{13} / \partial x_{1}$. Then a part of the Jacobi matrix is given by

$$
\left(\begin{array}{cccccc}
d_{1} & d_{2} & d_{3} & 0 & 0 & 0 \\
0 & 0 & 0 & d_{1} & d_{2} & d_{3}
\end{array}\right)
$$

where

$$
d_{1}=\operatorname{det}\left(\begin{array}{ll}
\frac{\partial g_{22}}{\partial x_{2}} & \frac{\partial g_{22}}{\partial y_{2}} \\
\frac{\partial g_{23}}{\partial x_{2}} & \frac{\partial g_{23}}{\partial y_{2}}
\end{array}\right), d_{2}=\operatorname{det}\left(\begin{array}{ll}
\frac{\partial g_{23}}{\partial x_{2}} & \frac{\partial g_{23}}{\partial y_{2}} \\
\frac{\partial g_{21}}{\partial x_{2}} & \frac{\partial g_{21}}{\partial y_{2}}
\end{array}\right), d_{3}=\operatorname{det}\left(\begin{array}{ll}
\frac{\partial g_{21}}{\partial x_{2}} & \frac{\partial g_{21}}{\partial y_{2}} \\
\frac{\partial g_{22}}{\partial x_{2}} & \frac{\partial g_{22}}{\partial y_{2}}
\end{array}\right) \text {. }
$$

Since ${ }_{2} j^{2}(\mu, g)(p) \in X, g_{2}$ is regular at $p$, above matrix is at least rank two. Hence $(0,0) \in \mathbb{R}^{2}$ is a regular value for $\zeta_{121122}$. Therefore $S_{21} \cap S_{22}$ is a closed submanifold of $X$ with codimension 2. $S_{23} \cap S_{24}$ is also a closed submanifold. Note that

$$
Y=X \backslash\left(\left(S_{21} \cap S_{22}\right) \cup\left(S_{23} \cap S_{24}\right)\right)
$$

are open submanifolds of $X$. If ${ }_{2} j^{2}(\mu, g) \in Y$, we have regular double point curves $c_{i j}$ : $(-\varepsilon, \varepsilon) \longrightarrow \mathbb{R}^{2}(i j=12,21)$ such that $g\left(c_{12}(t)\right)=g\left(c_{21}(t)\right)$ and $c_{i j}(0)=p_{i}$.

Moreover, the set

$$
S_{31}=\left\{{ }_{2} j^{2}(\mu, g)(p) \in Y \mid s_{31}=\frac{\partial \mu_{1}}{\partial x_{1}}\left(p_{1}\right) \cdot \Delta_{12}^{1}\left(p_{1}, p_{2}\right)+\frac{\partial \mu_{1}}{\partial y_{1}}\left(p_{1}\right) \cdot \Delta_{12}^{2}\left(p_{1}, p_{2}\right)=0\right\}
$$

and

$$
S_{32}=\left\{{ }_{2} j^{2}(\mu, g)(p) \in Y \mid s_{32}=\frac{\partial \mu_{2}}{\partial x_{2}}\left(p_{2}\right) \cdot \Delta_{21}^{1}\left(p_{1}, p_{2}\right)+\frac{\partial \mu_{2}}{\partial y_{2}}\left(p_{2}\right) \cdot \Delta_{21}^{2}\left(p_{1}, p_{2}\right)=0\right\}
$$


are closed submanifolds of $Y$. Finally, we put

$$
S_{41}=\left\{{ }_{2} j^{2}(\mu, g)(p) \in Y \mid s_{41}\left(p_{1}, p_{2}\right)=0\right\} \text { and } S_{42}=\left\{{ }_{2} j^{2}(\mu, g)(p) \in Y \mid s_{42}\left(p_{1}, p_{2}\right)=0\right\}
$$

where

$$
\begin{aligned}
& s_{41}=\frac{\partial^{2} \mu_{1}}{\partial x_{1}^{2}} \cdot\left(\Delta_{12}^{1}\right)^{2}+2 \frac{\partial^{2} \mu_{1}}{\partial x_{1} y_{1}} \cdot\left(\Delta_{12}^{1}\right) \cdot\left(\Delta_{12}^{2}\right)+\frac{\partial^{2} \mu_{1}}{\partial y_{1}^{2}} \cdot\left(\Delta_{12}^{2}\right)^{2}+\frac{\partial \mu_{1}}{\partial x_{1}} \cdot \Xi_{1}+\frac{\partial \mu_{1}}{\partial y_{1}} \cdot \Xi_{2}, \\
& s_{42}=\frac{\partial^{2} \mu_{2}}{\partial x_{2}^{2}} \cdot\left(\Delta_{21}^{1}\right)^{2}+2 \frac{\partial^{2} \mu_{2}}{\partial x_{2} y_{2}} \cdot\left(\Delta_{21}^{1}\right) \cdot\left(\Delta_{21}^{2}\right)+\frac{\partial^{2} \mu_{2}}{\partial y_{2}^{2}} \cdot\left(\Delta_{21}^{2}\right)^{2}+\frac{\partial \mu_{2}}{\partial x_{2}} \cdot \Xi_{3}+\frac{\partial \mu_{2}}{\partial y_{2}} \cdot \Xi_{4}, \\
& \Xi_{1}=-\operatorname{det}\left(\begin{array}{lll}
m_{21} & \partial g_{11} / \partial y_{1} & \partial g_{21} / \partial x_{2} \\
m_{22} & \partial g_{12} / \partial y_{1} & \partial g_{22} / \partial x_{2} \\
m_{23} & \partial g_{13} / \partial y_{1} & \partial g_{23} / \partial x_{2}
\end{array}\right), \Xi_{2}=-\operatorname{det}\left(\begin{array}{ccc}
\partial g_{11} / \partial x_{1} & m_{21} & \partial g_{21} / \partial x_{2} \\
\partial g_{12} / \partial x_{1} & m_{22} & \partial g_{22} / \partial x_{2} \\
\partial g_{13} / \partial x_{1} & m_{23} & \partial g_{23} / \partial x_{2}
\end{array}\right), \\
& \Xi_{3}=-\operatorname{det}\left(\begin{array}{lll}
m_{11} & \partial g_{21} / \partial y_{1} & \partial g_{21} / \partial x_{2} \\
m_{12} & \partial g_{22} / \partial y_{1} & \partial g_{22} / \partial x_{2} \\
m_{13} & \partial g_{23} / \partial y_{1} & \partial g_{23} / \partial x_{2}
\end{array}\right), \Xi_{4}=-\operatorname{det}\left(\begin{array}{ccc}
\partial g_{21} / \partial x_{1} & m_{11} & \partial g_{11} / \partial x_{2} \\
\partial g_{22} / \partial x_{1} & m_{12} & \partial g_{12} / \partial x_{2} \\
\partial g_{23} / \partial x_{1} & m_{13} & \partial g_{13} / \partial x_{2}
\end{array}\right), \\
& m_{1 j}=\frac{\partial^{2} g_{1 j}}{\partial x_{1}^{2}} \cdot\left(\Delta_{12}^{1}\right)^{2}+2 \frac{\partial^{2} g_{1 j}}{\partial x_{1} \partial y_{1}} \cdot\left(\Delta_{12}^{1}\right) \cdot\left(\Delta_{12}^{2}\right)+\frac{\partial^{2} g_{1 j}}{\partial y_{1}^{2}} \cdot\left(\Delta_{12}^{2}\right)^{2}-\frac{\partial^{2} g_{2 j}}{\partial x_{2}^{2}} \cdot\left(\Delta_{21}^{1}\right)^{2} \\
& -2 \frac{\partial^{2} g_{2 j}}{\partial x_{2} \partial y_{2}} \cdot\left(\Delta_{21}^{1}\right) \cdot\left(\Delta_{21}^{2}\right)-\frac{\partial^{2} g_{2 j}}{\partial y_{2}^{2}} \cdot\left(\Delta_{21}^{2}\right)^{2}
\end{aligned}
$$

and

$$
\begin{array}{r}
m_{2 j}=\frac{\partial^{2} g_{2 j}}{\partial x_{2}^{2}} \cdot\left(\Delta_{21}^{1}\right)^{2}+2 \frac{\partial^{2} g_{2 j}}{\partial x_{2} \partial y_{2}} \cdot\left(\Delta_{21}^{1}\right) \cdot\left(\Delta_{21}^{2}\right)+\frac{\partial^{2} g_{2 j}}{\partial y_{2}^{2}} \cdot\left(\Delta_{21}^{2}\right)^{2}-\frac{\partial^{2} g_{2 j}}{\partial x_{1}^{2}} \cdot\left(\Delta_{12}^{1}\right)^{2} \\
-2 \frac{\partial^{2} g_{2 j}}{\partial x_{1} \partial y_{1}} \cdot\left(\Delta_{12}^{1}\right) \cdot\left(\Delta_{12}^{2}\right)-\frac{\partial^{2} g_{2 j}}{\partial y_{1}^{2}} \cdot\left(\Delta_{12}^{2}\right)^{2}
\end{array}
$$

Then subsets $S_{31} \cap S_{41}$ and $S_{32} \cap S_{42}$ are submanifolds of $Y$ with codimension 2. Indeed, we define a function $s: Y \longrightarrow \mathbb{R}^{2}$ by $s\left({ }_{2} j^{2}(\mu, g)\left(x_{1}, y_{1} ; x_{2}, y_{2}\right)\right)=\left(s_{31}\left(x_{1}, y_{1} ; x_{2}, y_{2}\right), s_{41}\left(x_{1}, y_{1} ; x_{2}, y_{2}\right)\right)$ then the derivative of $s$ with respect to the coordinates of $Y$ corresponding to the derivatives $\partial \mu_{1} / \partial x_{1}, \partial \mu_{1} / \partial y_{1}, \partial \mu_{2} / \partial x_{2}$ and $\partial^{2} \mu_{2} / \partial y_{1}^{2}$ is given by

$$
\left(\begin{array}{cccc}
\Delta_{12}^{1} & \Delta_{12}^{2} & 0 & 0 \\
* & * & \left(\Delta_{12}^{1}\right)^{2} & \left(\Delta_{12}^{2}\right)^{2}
\end{array}\right) .
$$

Thus $(0,0) \in \mathbb{R}^{2}$ is a regular value of $s$ and $s^{-1}(0,0)=S_{31} \cap S_{41}$ is a submanifold with codimension 2. By the same method, $S_{32} \cap S_{42}$ is a submanifold with codimension 2.

We put $S_{3}=S_{21} \cap S_{22}, S_{4}=S_{23} \cap S_{24}, S_{5}=S_{31} \cap S_{32}, S_{6}=S_{31} \cap S_{41}, S_{7}=S_{32} \cap S_{42}$, and $\widetilde{S}_{*}=\mathbb{R}^{2^{(2)}} \times \mathbb{R} \times \mathbb{R} \times{ }_{2} \Delta \times S_{*}$, where $*=1,2,3,4,5,6$ and 7 .

Since all $\widetilde{S}_{*}$ are invariant sets with respect to the equivalence of divergent diagrams, we can extend to submanifold $\overline{S_{*}}$ in the multi-jet space ${ }_{2} J^{2}(M, P \times N)$ by the same way of $r=1$. From the multi-jet transversality theorem, the set

$$
\begin{array}{r}
\mathcal{O}_{2}=\left\{\left.(\mu, g) \in C^{\infty}(M, N \times P)\right|_{2} j^{2}(\mu, g)\right. \text { is transverse to } \\
\left.W^{2}, \overline{S_{1}}, \overline{S_{2}}, \overline{S_{3}}, \overline{S_{4}}, \overline{S_{5}}, \overline{S_{6}} \text { and } \overline{S_{7}}\right\}
\end{array}
$$


is a residual subset of $C^{\infty}(M, N \times P)$ with respect to the Whitney $C^{\infty}$-topology, where $W^{2}$ is that of Proposition 2.2. Remark that from $\operatorname{dim} \mathbb{R}^{2^{(2)}}=4$ and $\operatorname{codim} \overline{S_{*}} \geq 5,{ }_{2} j^{2}(\mu, g)$ is transverse to $\overline{S_{*}}$ is equivalent to ${ }_{2} j^{2}(\mu, g)\left(\mathbb{R}^{2(2)}\right) \cap \overline{S_{*}}=\emptyset(*=1,2,3,4,5,6$ and 7$)$.

Now we show that $(\mu, g) \in \mathcal{O}_{2}$ means the condition of lemma. Suppose that $(\mu, g) \in \mathcal{O}_{2}$. Then we put

$$
D\left(p_{1}, p_{2}\right)=\left\{\left(x_{1}, y_{1} ; x_{2}, y_{2}\right) \in U_{1} \times U_{2} \mid g_{1}\left(x_{1}, y_{1}\right)-g_{2}\left(x_{2}, y_{2}\right)=0\right\}
$$

Since ${ }_{2} j^{2}(\mu, g)\left(\mathbb{R}^{2(2)}\right) \cap\left(\overline{S_{1}} \cup \overline{S_{2}}\right)=\emptyset, g_{1}$ and $g_{2}$ are immersion germs and $D\left(p_{1}, p_{2}\right)$ is a certain regular curve in $U_{1} \times U_{2}$. $D\left(p_{1}, p_{2}\right)$ characterizes the double point curves lie on the source space. We take a parameterization $\gamma(t)=\left(c_{12}(t), c_{21}(t)\right)=\left(\left(c_{12}^{1}(t), c_{12}^{2}(t)\right),\left(c_{21}^{1}(t), c_{21}^{2}(t)\right)\right)$ of $D\left(p_{1}, p_{2}\right)$, then $c_{12}$ and $c_{21}$ are regular curves.

We now show transversality conditions implies that both of $\mu_{1} \circ c_{12}$ and $\mu_{2} \circ c_{21}$ are immersion germs, or one of $\mu_{1} \circ c_{12}$ and $\mu_{2} \circ c_{21}$ is an immersion germ and the another is Morse function germ.

By the implicit function theorem, we have

$$
\frac{d\left(\mu_{1} \circ c_{12}\right)}{d t}=\lambda_{1} \cdot\left(\frac{\partial \mu_{1}}{\partial x_{1}} \cdot \Delta_{12}^{1}+\frac{\partial \mu_{1}}{\partial y_{1}} \cdot \Delta_{12}^{2}\right), \frac{d\left(\mu_{2} \circ c_{21}\right)}{d t}=\lambda_{2} \cdot\left(\frac{\partial \mu_{2}}{\partial x_{2}} \cdot \Delta_{21}^{1}+\frac{\partial \mu_{2}}{\partial y_{2}} \cdot \Delta_{21}^{2}\right),
$$

where $\lambda_{1}$ and $\lambda_{2}$ are non-zero functions around $p_{1}$ and $p_{2}$ respectively.

Under the condition $d\left(\mu_{1} \circ c_{12}\right) / d t=0$ and $d\left(\mu_{2} \circ c_{21}\right) / d t=0$, we have

$\frac{d^{2}\left(\mu_{1} \circ c_{12}\right)}{d t^{2}}=\lambda_{3} \cdot\left(\frac{\partial^{2} \mu_{1}}{\partial x_{1}^{2}} \cdot\left(\Delta_{12}^{1}\right)^{2}+2 \frac{\partial^{2} \mu_{1}}{\partial x_{1} \partial y_{1}} \cdot\left(\Delta_{12}^{1}\right) \cdot\left(\Delta_{12}^{2}\right)+\frac{\partial^{2} \mu_{1}}{\partial y_{1}^{2}} \cdot\left(\Delta_{12}^{2}\right)^{2}+\frac{\partial \mu_{1}}{\partial x_{1}} \cdot \Xi_{1}+\frac{\partial \mu_{1}}{\partial y_{1}} \cdot \Xi_{2}\right)$

and

$\frac{d^{2}\left(\mu_{2} \circ c_{21}\right)}{d t^{2}}=\lambda_{4} \cdot\left(\frac{\partial^{2} \mu}{\partial x_{2}^{2}} \cdot\left(\Delta_{21}^{1}\right)^{2}+2 \frac{\partial^{2} \mu_{2}}{\partial x_{2} \partial y_{2}} \cdot\left(\Delta_{21}^{1}\right) \cdot\left(\Delta_{21}^{2}\right)+\frac{\partial^{2} \mu_{2}}{\partial y_{2}^{2}} \cdot\left(\Delta_{21}^{2}\right)^{2}+\frac{\partial \mu_{2}}{\partial x_{2}} \cdot \Xi_{3}+\frac{\partial \mu_{2}}{\partial y_{2}} \cdot \Xi_{4}\right)$,

where $\lambda_{3}$ and $\lambda_{4}$ are non-zero functions around $p_{1}$ and $p_{2}$ respectively. Therefore

$$
{ }_{2} j^{2}(\mu, g)\left(\mathbb{R}^{2(2)}\right) \cap\left(\overline{S_{1}} \cup \overline{S_{2}} \cup \overline{S_{3}} \cup \overline{S_{4}} \cup \overline{S_{5}} \cup \overline{S_{6}} \cup \overline{S_{7}}\right)=\emptyset
$$

implies the conditions of lemma.

In the case of $r=3$. We put $g^{-1}(g(p))=\left\{p_{1}, p_{2}, p_{3}\right\}$. Under this notation, we have the following lemma.

Lemma 4.2. There exists a residual subset $\mathcal{O}_{3} \subset C^{\infty}(M, P \times N)$ such that for any $(\mu, g) \in \mathcal{O}_{3}$ and $p \in M$, if $\sharp g^{-1}(g(p))=3$, then map germs $(\mu, g)$ at $p_{1}, p_{2}$ and $p_{3}$ satisfy the conditions of (I,I,I) of Theorem 3.1. 
Proof. We take open neighborhoods $U_{i}$ at $p_{i}$ and local coordinates $\left(x_{i}, y_{i}\right)$ on $U_{i}(i=1,2,3)$. Denote $g_{i}=\left(g_{i 1}, g_{i 2}, g_{i 3}\right)$ by map germs $g$ at $p_{i}$ with respect to the coordinates. By the same way of proof of Lemma 4.1, first we put

$$
S_{i}=\left\{{ }_{3} j^{2}(\mu, g)(p) \in{ }_{3} J^{2}(2,4)_{(p)} \mid d g_{i}\left(p_{i}\right)=0\right\}(i=1,2,3) .
$$

Then $\cup_{i=1,2,3} S_{i}$ is a closed algebraic subset with codimension 2. Note that

$$
X={ }_{3} J^{2}(2,4)_{(p)} \backslash \bigcup_{i=1,2,3} S_{i}
$$

is an open submanifold of ${ }_{3} J^{2}(2,4)_{(p)}$. Second, we put

$$
S_{i j}=\left\{{ }_{3} j^{2}(\mu, g)(p) \in X \mid \Delta_{i j}^{1}\left(p_{1}, p_{2}, p_{3}\right)=\Delta_{i j}^{2}\left(p_{1}, p_{2}, p_{3}\right)=0\right\}
$$

and define maps $\zeta_{i j}: X \longrightarrow \mathbb{R}^{2}(i j=12,21,13,31,23$ or 32$)$ by

$$
\zeta_{i j}\left({ }_{3} j^{2}(\mu, g)\left(x_{1}, y_{1} ; x_{2}, y_{2} ; x_{3}, y_{3}\right)\right)=\left(\Delta_{i j}^{1}\left(x_{1}, y_{1} ; x_{2}, y_{2} ; x_{3}, y_{3}\right), \Delta_{i j}^{2}\left(x_{1}, y_{1} ; x_{2}, y_{2} ; x_{3}, y_{3}\right)\right)
$$

Then $S_{i j}=\zeta_{i j}^{-1}(0,0)$ and we see that $(0,0) \in \mathbb{R}^{2}$ is a regular value for $\zeta_{i j}$. Therefore $S_{i j}$ are closed submanifolds of $X$ with codimension 2. Note that

$$
Y=X \backslash \bigcup_{i j=12,21,13,31,23,32} S_{i j}
$$

is an open submanifold of $X$.

We remember if ${ }_{3} j^{2}(\mu, g) \in Y$, then double points form regular curves $c_{i j}(t):(-\varepsilon, \varepsilon) \longrightarrow \mathbb{R}^{2}$ $(i j=12,21,13,31,23,32)$ such that $g\left(c_{i j}(t)\right)=g\left(c_{j i}(t)\right)$ and $c_{i j}(0)=p_{i}$.

Third, we put

$$
S_{3 i j}=\left\{\begin{array}{l|l|l}
{ }_{3} j^{2}(\mu, g) \in Y & \frac{\partial \mu_{i}}{\partial x_{i}} \cdot \Delta_{i j}^{1}+\frac{\partial \mu_{i}}{\partial y_{i}} \cdot \Delta_{i j}^{2}=0
\end{array}\right\}
$$

Then $S_{3 i j}(i j=12,21,13,31,23$ and 32$)$ are closed submanifold of $Y$ with codimension 1 . We also put $\widetilde{S_{*}}=\mathbb{R}^{2^{(3)}} \times \mathbb{R} \times \mathbb{R} \times \mathbb{R} \times{ }_{3} \Delta \times S_{*}$ and we have the submanifolds $\overline{S_{i}}, \overline{S_{i j}}$ and $\overline{S_{3 i j}}$ in multi-jet space ${ }_{3} J^{2}(M, N \times P)$ by the same way of $r=1$.

From multi-jet transversality theorem, the set

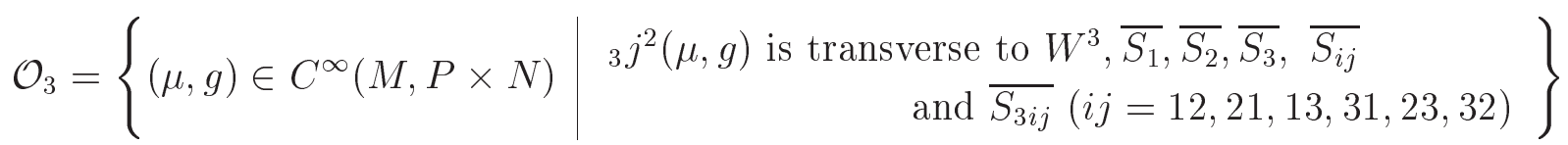

is a residual subset, where $W^{3}$ defined in Proposition 2.2. Since $\operatorname{dim} \mathbb{R}^{2^{(3)}}=6$ and $\operatorname{codim} \overline{S_{*}} \geq 7$, we have that ${ }_{3} j^{2}(\mu, g)$ is transverse to $\overline{S_{*}}$ is equivalent to ${ }_{3} j^{2}(\mu, g)\left(\mathbb{R}^{2^{(3)}}\right) \cap \overline{S_{*}}=\emptyset$.

We can show that $(\mu, g) \in \mathcal{O}_{3}$ means the conditions of lemma by the same method of proof of Lemma 4.1 .

By Lemmas 4.1 and 4.2, if we put $\mathcal{O}=\mathcal{O}_{1} \cap \mathcal{O}_{2} \cap \mathcal{O}_{3}$, then we proof the Theorem 3.1. 


\section{Proof of Theorem 3.2}

In the case of $r=1$. For types (I) and (II), by the diffeomorphisms of domain and target and by Morse's lemma, we obtain the normal forms (I) and (II) $)_{ \pm}$in Theorem 3.2.

We consider the type (III). We denote by $(x, y)$ the local coordinate at $p$ and $(u, v, w)$ the local coordinate at $g(p)$. Let $\mu:\left(\mathbb{R}^{2}, 0\right) \longrightarrow(\mathbb{R}, 0)$ be a submersion germ, $g:\left(\mathbb{R}^{2}, 0\right) \longrightarrow\left(\mathbb{R}^{3}, 0\right)$ be a Whitney umbrella and $(\mu, g)$ be an immersion germ. By the normal form of the Whitney umbrella, we may assume that $g(x, y)=\left(x^{2}, y, x y\right)$.

We need the following two obvious lemmas:

Lemma 5.1. Let $g=\left(x^{2}, y, x y\right):\left(\mathbb{R}^{2}, 0\right) \longrightarrow\left(\mathbb{R}^{3}, 0\right)$ be a Whitney umbrella germ and let $H:\left(\mathbb{R}^{2}, 0\right) \longrightarrow\left(\mathbb{R}^{2}, 0\right)$ and $K:\left(\mathbb{R}^{3}, 0\right) \longrightarrow\left(\mathbb{R}^{3}, 0\right)$ be diffeomorphism germs defined by

$$
H=(x \cdot \alpha \circ g, y \cdot \beta \circ g) \text { and } K=\left(u \cdot \alpha^{2}, v \cdot \beta, w \cdot \alpha \cdot \beta\right) \text {, }
$$

where $\alpha, \beta \in \mathcal{E}_{(u, v, w)}$ with $\alpha(0) \neq 0, \beta(0) \neq 0$. Then $g \circ H=K \circ g$ holds.

Lemma 5.2. Let $g=\left(x^{2}, y, x y\right):\left(\mathbb{R}^{2}, 0\right) \longrightarrow\left(\mathbb{R}^{3}, 0\right)$ be a Whitney umbrella germ and $\alpha:$ $\left(\mathbb{R}^{3}, 0\right) \longrightarrow(\mathbb{R}, 0)$ be a function germ given by $\alpha(u, v, w)=v \beta(v)$, where $\beta \in \mathcal{E}_{v}$. We define diffeomorphism germs $H:\left(\mathbb{R}^{2}, 0\right) \longrightarrow\left(\mathbb{R}^{2}, 0\right)$ and $K:\left(\mathbb{R}^{3}, 0\right) \longrightarrow\left(\mathbb{R}^{3}, 0\right)$ by

$$
H=(x+\alpha \circ g, y) \text { and } K=\left(u+2 w \cdot \beta+\alpha^{2}, v, w+v \cdot \alpha\right) \text {. }
$$

Then $g \circ H=K \circ g$ holds.

We also need the following lemma:

Lemma 5.3. Let $g=\left(x^{2}, y, x y\right):\left(\mathbb{R}^{2}, 0\right) \longrightarrow\left(\mathbb{R}^{3}, 0\right)$ be a Whitney umbrella germ and let $H:\left(\mathbb{R}^{2}, 0\right) \longrightarrow\left(\mathbb{R}^{2}, 0\right)$ be diffeomorphism germs defined by $H(x, y)=(x, y \cdot \phi(x, y))$, where $\phi \in \mathcal{E}_{(x, y)}$ and $\phi(0) \neq 0$. Then there exists a diffeomorphism germ $K:\left(\mathbb{R}^{3}, 0\right) \longrightarrow\left(\mathbb{R}^{3}, 0\right)$ such that $K \circ g=g \circ H$.

Proof. We calculate that $g \circ H(x, y)=\left(x^{2}, y \cdot \phi(x, y), x y \cdot \phi(x, y)\right)$. By the Malgrange preparation theorem, there exist function germs $k, h \in \mathcal{E}_{(u, v, w)}$ such that $y \cdot \phi(x, y)=k \circ g(x, y)$ and $x y \cdot \phi(x, y)=h \circ g(x, y)$. Since $\phi(0) \neq 0$, we have $(\partial k / \partial v)(0) \neq 0$ and $(\partial h / \partial w)(0) \neq 0$. Therefore we define a diffeomorphism germ $K:\left(\mathbb{R}^{3}, 0\right) \longrightarrow\left(\mathbb{R}^{3}, 0\right)$ by

$$
K(u, v, w)=(u, k(u, v, w), h(u, v, w)),
$$

then it is clear that the condition $K \circ g=g \circ H$ holds.

We now detect a normal form of type (III). Since $(\mu, g)$ is an immersion germ and $g(x, y)=$ $\left(x^{2}, y, x y\right), \mu$ is satisfied the condition $(\partial \mu / \partial x)(0) \neq 0$. Applying the Malgrange preparation theorem, we have

$$
\mu=x \cdot \alpha \circ g+\beta \circ g
$$


where $\alpha, \beta \in \mathcal{E}_{(u, v, w)}$ and $\alpha(0) \neq 0$. Thus we can define a coordinate transformation $H$ and $K$ by

$$
H=(x \cdot \alpha \circ g, y) \text { and } K=\left(u \cdot \alpha^{2}, v, w \cdot \alpha\right) .
$$

Taking new coordinates by $H, K$ from Lemma $5.1,(\mu, g)$ is equivalent to $\left(\mu^{\prime}, g\right)$ which is given by

$$
\mu^{\prime}(x, y)=x+\beta \circ g(x, y), g(x, y)=\left(x^{2}, y, x y\right)
$$

By Lemma 5.2, if necessary, consider the diffeomorphism germ $H(x, y)=(x-y, y)$, then $\left(\mu^{\prime}, g\right)$ is equivalent to $\left(\mu^{\prime \prime}, g\right)$ which is given by

$$
\mu^{\prime \prime}(x, y)=x+\gamma \circ g(x, y)+y, g(x, y)=\left(x^{2}, y, x y\right)
$$

where $\gamma \in \mathcal{M}_{(u, v, w)}$. Finally we decompose $\mu^{\prime \prime}(x, y)$ into $x+\widetilde{\alpha}\left(x^{2}\right)+y \cdot \widetilde{\beta}(x, y)$, where $\widetilde{\alpha} \in$ $\mathcal{E}_{x}, \widetilde{\beta} \in \mathcal{E}_{(x, y)}$ and $\widetilde{\beta}(0) \neq 0$. By Lemma 5.3, we consider the diffeomorphism germ $H(x, y)=$ $(x, y \cdot \widetilde{\beta}(x, y))$. Then we obtain the normal form of type (III) in Theorem 3.2.

In the case of $r=2$. Let $(\mu, g)$ be type of $(\mathrm{I}, \mathrm{I})_{1}$ or $(\mathrm{I}, \mathrm{I})_{2}$. We denote germs of $(\mu, g)$ at $p_{1}$ and $p_{2}$ by $\left(\mu_{1}, g_{1}\right)$ and $\left(\mu_{2}, g_{2}\right)$ respectively. In this case, we take local coordinates $(x, y)$ at $p_{1}$, $(u, v)$ at $p_{2}$ and $(p, q, r)$ at $g_{1}\left(p_{1}\right)=g_{2}\left(p_{2}\right)$.

Since image of $g_{1}$ and $g_{2}$ are general position, we may assume that $g_{1}(x, y)=(x, y, 0)$ and $g_{2}(u, v)=(0, u, v)$. By the condition of $(\mathrm{I}, \mathrm{I})_{1}$ and $(\mathrm{I}, \mathrm{I})_{2},\left(\mu_{1}, g_{1}\right)$ and $\left(\mu_{2}, g_{2}\right)$ are satisfied one of the following condition:

(1) $\frac{\partial \mu_{1}}{\partial y}(0) \cdot \frac{\partial \mu_{2}}{\partial u}(0) \neq 0$

(2) $\frac{\partial \mu_{1}}{\partial y}(0)=0, \frac{\partial^{2} \mu_{1}}{\partial y^{2}}(0) \neq 0$ and $\frac{\partial \mu_{1}}{\partial x}(0) \cdot \frac{\partial \mu_{2}}{\partial u}(0) \neq 0$.

For the case of (1), we consider the diffeomorphism germs

$$
\phi_{1}:\left(\mathbb{R}^{2}, 0\right) \longrightarrow\left(\mathbb{R}^{2}, 0\right) \text { and } \Psi:\left(\mathbb{R}^{3}, 0\right) \longrightarrow\left(\mathbb{R}^{3}, 0\right)
$$

given by $\phi_{1}(x, y)=\left(x, \mu_{1}(x, y)\right)$ and $\Psi(p, q, r)=\left(p, \mu_{1}(p, q), r\right)$. Then the pair of divergent diagrams $\left(\mu_{1}, g_{1} ; \mu_{2}, g_{2}\right)$ is equivalent to

$$
\left(\mu_{1}, g_{1}\right)=(y, x, y, 0),\left(\mu_{2}, g_{2}\right)=\left(\mu_{2}(u, v), 0, \mu_{1}(0, u), v\right)
$$

Furthermore we consider a coordinate transformation

$$
\phi_{1}:\left(\mathbb{R}^{2}, 0\right) \longrightarrow\left(\mathbb{R}^{2}, 0\right), \phi_{2}:\left(\mathbb{R}^{2}, 0\right) \longrightarrow\left(\mathbb{R}^{2}, 0\right), \Psi:\left(\mathbb{R}^{3}, 0\right) \longrightarrow\left(\mathbb{R}^{3}, 0\right)
$$

and

$$
\kappa_{1}:(\mathbb{R}, 0) \longrightarrow(\mathbb{R}, 0)
$$

given by

$$
\phi_{1}(x, y)=\left(x, \mu_{2}\left(\mu_{1}^{-1}(0, y), 0\right)\right), \phi_{2}(u, v)=\left(\mu_{2}(u, v), v\right), \Psi(p, q, r)=\left(p, \mu_{2}\left(\mu_{1}^{-1}(0, q), r\right), r\right)
$$


and

$$
\kappa_{1}(s)=\mu_{2}\left(\mu_{1}^{-1}(0, s), 0\right) .
$$

Hence, taking new coordinates by the above diffeomorphisms, the pair of divergent diagrams $\left(\mu_{1}, g_{1} ; \mu_{2}, g_{2}\right)$ is equivalent to

$$
\left(\mu_{1}, g_{1}\right)=(y, x, y, 0),\left(\mu_{2}, g_{2}\right)=(u, 0, u, v)
$$

For the case of (2), we consider the diffeomorphism germs

$$
\phi_{2}:\left(\mathbb{R}^{2}, 0\right) \longrightarrow\left(\mathbb{R}^{2}, 0\right) \text { and } \Psi:\left(\mathbb{R}^{3}, 0\right) \longrightarrow\left(\mathbb{R}^{3}, 0\right)
$$

given by $\phi_{2}(u, v)=\left(\mu_{2}(u, v), v\right)$ and $\Psi(p, q, r)=\left(p, \mu_{2}(q, r), r\right)$. Then the pair of divergent diagrams $\left(\mu_{1}, g_{1} ; \mu_{2}, g_{2}\right)$ is equivalent to

$$
\left(\mu_{1}, g_{1}\right)=\left(\mu_{1}(x, y), x, \mu_{2}(y, 0), 0\right),\left(\mu_{2}, g_{2}\right)=(u, 0, u, v)
$$

We also define a coordinate transformation

$$
\phi_{1}:\left(\mathbb{R}^{2}, 0\right) \longrightarrow\left(\mathbb{R}^{2}, 0\right) \text { and } \Psi:\left(\mathbb{R}^{3}, 0\right) \longrightarrow\left(\mathbb{R}^{3}, 0\right)
$$

given by $\phi_{1}(x, y)=\left(\mu_{1}(x, y), \mu_{2}(y, 0)\right)$ and $\left.\Psi(p, q, r)=\left(\mu_{1}\left(p, \mu_{2}^{-1}(q, 0), r\right), q, r\right)\right)$. Taking new coordinates by above diffeomorphisms, the pair of divergent diagrams $\left(\mu_{1}, g_{1} ; \mu_{2}, g_{2}\right)$ is equivalent to

$$
\left(\mu_{1}, g_{1}\right)=(y, x, y, 0),\left(\mu_{2}, g_{2}\right)=\left(v, \mu_{1}\left(0, \mu_{2}^{-1}(u, 0)\right), u, v\right) .
$$

Moreover, we put $\nu(y)=\mu_{1}\left(0, \mu_{2}^{-1}(y, 0)\right)$ and consider the coordinate transformation

$$
\phi_{1}:\left(\mathbb{R}^{2}, 0\right) \longrightarrow\left(\mathbb{R}^{2}, 0\right) \text { and } \Psi:\left(\mathbb{R}^{3}, 0\right) \longrightarrow\left(\mathbb{R}^{3}, 0\right)
$$

given by $\phi_{1}(x, y)=(x-\nu(y), y)$ and $\Psi(p, q, r)=(p-\nu(q), q, r)$, then the pair of divergent diagrams $\left(\mu_{1}, g_{1} ; \mu_{2}, g_{2}\right)$ is equivalent to

$$
\left(\mu_{1}, g_{1}\right)=(x+\nu(y), x, y, 0),\left(\mu_{2}, g_{2}\right)=(u, 0, u, v)
$$

By the condition $\left(\partial^{2} \mu_{1} / \partial y^{2}\right)(0) \neq 0$, we have $\left(d^{2} \nu / d y^{2}\right)(0) \neq 0$. Therefore there exists a diffeomorphism germ $\psi:(\mathbb{R}, 0) \longrightarrow(\mathbb{R}, 0)$ such that $\nu \circ \psi^{-1}(y)=y^{2}$. Then we consider a coordinate transformation

$$
\phi_{1}:\left(\mathbb{R}^{2}, 0\right) \longrightarrow\left(\mathbb{R}^{2}, 0\right), \phi_{2}:\left(\mathbb{R}^{2}, 0\right) \longrightarrow\left(\mathbb{R}^{2}, 0\right), \Psi:\left(\mathbb{R}^{3}, 0\right) \longrightarrow\left(\mathbb{R}^{3}, 0\right)
$$

and

$$
\kappa_{2}:(\mathbb{R}, 0) \longrightarrow(\mathbb{R}, 0)
$$

given by

$$
\phi_{1}(x, y)=(x, \psi(y)), \phi_{2}(u, v)=(\psi(u), v), \Psi(p, q, r)=(p, \psi(q), r)
$$


and

$$
\kappa_{2}(s)=\psi(s) .
$$

Therefore the pair of divergent diagrams $\left(\mu_{1}, g_{1} ; \mu_{2}, g_{2}\right)$ is equivalent to

$$
\left(\mu_{1}, g_{1}\right)=\left(x+y^{2}, x, y, 0\right),\left(\mu_{2}, g_{2}\right)=(u, 0, u, v) .
$$

In the case of $r=3$. Let $(\mu, g)$ be type (I,I,I) and $\left(\mu_{i}, g_{i}\right)$ be germs at $p_{i}(i=1,2,3)$. In this case, we also take local coordinates $(x, y)$ at $p_{1},(u, v)$ at $p_{2},(a, b)$ at $p_{3}$ and $(p, q, r)$ at $g_{1}\left(p_{1}\right)=g_{2}\left(p_{2}\right)=g_{3}\left(p_{3}\right)$.

By the condition (I,I,I) and the case of $r=2,\left(\mu_{1}, g_{1} ; \mu_{2}, g_{2} ; \mu_{3}, g_{3}\right)$ is equivalent to

$$
\left(\mu_{1}, g_{1}\right)=(y, x, y, 0),\left(\mu_{2}, g_{2}\right)=(u, 0, u, v),\left(\mu_{3}, g_{3}\right)=\left(\mu_{3}(a, b), a, h(a, b), b\right),
$$

where $h \in \mathcal{M}_{(a, b)}$. We apply a coordinate transformation

$$
\phi_{1}:\left(\mathbb{R}^{2}, 0\right) \longrightarrow\left(\mathbb{R}^{2}, 0\right), \phi_{2}:\left(\mathbb{R}^{2}, 0\right) \longrightarrow\left(\mathbb{R}^{2}, 0\right) \text { and } \Psi:\left(\mathbb{R}^{3}, 0\right) \longrightarrow\left(\mathbb{R}^{3}, 0\right)
$$

given by $\phi_{1}(x, y)=(x, y-h(x, 0)), \phi_{2}(u, v)=(u-h(0, v), v)$ and $\Psi(p, q, r)=(p, q-h(p, r), r)$, then the divergent diagrams $\left(\mu_{1}, g_{1} ; \mu_{2}, g_{2} ; \mu_{3}, g_{3}\right)$ is equivalent to

$$
\left(\mu_{1}, g_{1}\right)=(y+h(x, 0), x, y, 0),\left(\mu_{2}, g_{2}\right)=(u+h(0, v), 0, u, v),\left(\mu_{3}, g_{3}\right)=\left(\mu_{3}(a, b), a, 0, b\right),
$$

where $h(a, b) \in \mathcal{M}_{(a, b)}$. Again by the condition (I,I,I), then the function germs $h$ and $\mu_{3}$ satisfy the condition

$$
\frac{\partial h}{\partial a}(0) \neq 0, \frac{\partial h}{\partial b}(0) \neq 0, \frac{\partial \mu_{3}}{\partial a}(0) \neq 0 \text { and } \frac{\partial \mu_{3}}{\partial b}(0) \neq 0 .
$$

Finally, we consider a coordinate transformation

$$
\phi_{1}:\left(\mathbb{R}^{2}, 0\right) \longrightarrow\left(\mathbb{R}^{2}, 0\right), \phi_{2}:\left(\mathbb{R}^{2}, 0\right) \longrightarrow\left(\mathbb{R}^{2}, 0\right), \phi_{3}:\left(\mathbb{R}^{2}, 0\right) \longrightarrow\left(\mathbb{R}^{2}, 0\right)
$$

and

$$
\Psi:\left(\mathbb{R}^{3}, 0\right) \longrightarrow\left(\mathbb{R}^{3}, 0\right)
$$

given by

$$
\phi_{1}(x, y)=(h(x, 0), y), \phi_{2}(u, v)=(u, h(0, v)), \phi_{3}(a, b)=(h(a, 0), h(0, b))
$$

and

$$
\Psi(p, q, r)=(h(p, 0), q, h(0, r)),
$$

then the divergent diagrams $\left(\mu_{1}, g_{1} ; \mu_{2}, g_{2} ; \mu_{3}, g_{3}\right)$ is equivalent to

$$
\left(\mu_{1}, g_{1}\right)=(x+y, x, y, 0),\left(\mu_{2}, g_{2}\right)=(u+v, 0, u, v),\left(\mu_{3}, g_{3}\right)=\left(\mu_{3}(a, b), a, 0, b\right),
$$

where $\left(\partial \mu_{3} / \partial a\right)(0) \neq 0$ and $\left(\partial \mu_{3} / \partial b\right)(0) \neq 0$ Therefore we obtain the normal form of (I,I,I) in Theorem 3.2.

This complete proof of the Theorem 3.2. 


\section{References}

[1] V.I. Arnol'd, Wave front evolution and equivalent Morse lemma. Comm. Pure Appl. Math., 29 (1976), 557-582.

[2] D. Bleecker and L. Wilson, Stability of Gauss maps. Illinois J. Math., 22 (1978), 279-289.

[3] J. Boardman, Singularities of differentiable maps. Publ. Math. I.H.E.S., 33 (1967), 21-57.

[4] J.W. Bruce, Seeing-the Mathematical viewpoint. Math. Intelligencer, 6 No.4. (1984), 18-25.

[5] J.P. Dufour, Families de courbes planes différentiables. Topology, 22 (1983), 449-474.

[6] J.P. Dufour, Modules pour les familles de courbes planes. Ann. Inst. Fourier, Grenoble. 39 (1989), 225-238.

[7] J.P. Dufour and P. Jean, Rigidity of webs and families of hypersurfaces. Singularities and dynamical systems (ed. S.N. Pnevmatikos, Elsevier/ North-Holland, Amsterdam) (1985), 271283.

[8] J.P. Dufour and P. Jean, Familles de surfaces differentiables. J. London Math. Soc. (2) 42 (1990), $175-192$.

[9] J.P. Dufour and Y. Kurokawa, Topological rigidity for certain families of differentiable plane curves. J. London Math. Soc., 65 (2002), 223-242.

[10] J.P. Dufour and J.P. Tueno, Singularitiés et photographies de surfaces. J. Geom. Phys. 9 (1992), $173-182$.

[11] C.G. Gibson, Singular Points of Smooth Mappings. Pitman, London, 1979.

[12] M. Golubitsky and V. Guillemin, Stable Mappings and their Singularities. Graduate Texts in Math. 14, Springer-Verlag, New York, 1973.

[13] S. Izumiya and Y. Kurokawa, Holonomic systems of Clairaut type. Diff. Geometry and App. 5 (1995), 219-235.

[14] S. Izumiya and W.L. Marar, On topologically stable singular surfaces in a 3-manifold. J. of Geom., 52 (1995), 108-119.

[15] S. Izumiya and K. Maruyama, Transversal Whitney topology and singularities of Haefliger foliations. Lecture Note in Pure and Appl. Math., 232 (2003), 165-173.

[16] Y. Kurokawa, Singularities for projections of contour lines of surfaces onto planes. Hokkaido Math. J. 30 (2001), 573-587.

[17] S. Mancini, M.A.S. Ruas and M.A. Teixeira, On divergent diagrams of finite codimension. Portugaliae Math. 59 (2002), 179-194. 
[18] J. Mather, Stability of $C^{\infty}$-mappings V. Transversality. Adv. in Math. 4 (1970), 301-336.

[19] T. Nishimura, A constructive method to get right-left equivalence for smooth map germs and its application to divergent diagrams. Workshop on Real and Complex Singularities, Mat. Contemp. 5 (1993), 137-160.

[20] M. Takahashi, Holonomic systems of general Clairaut type. Hokkaido Math. J. 34 (2005), 247263.

[21] C.T.C. Wall, Openness and Multitransversality. Lecture Note in Pure and Appl. Math., 232 (2003), 121-136.

[22] H. Whitney, The singularities of smooth $n$-manifold in (2n-1)-space. Annals of Math. 45 (1944), $247-293$.

[23] H. Whitney, Elementary structure of real algebraic varieties. Annals of Math. 66 (1957), 545-556.

Kentaro Saji, Department of Mathematics, Hokkaido University, Sapporo 060-0810, JAPAN. E-mail: saji@math.sci.hokudai.ac.jp

Masatomo Takahashi, Department of Mathematics, Hokkaido University, Sapporo 060-0810, JAPAN. E-mail: takahashi@math.sci.hokudai.ac.jp 